\section{ALPHABET SOUP}

Jean Paul Revel, Ph.D.

CALTECH, Pasadena, CA

I have just attended the 50th anniversary of EMSA in Boston, and what a grand show it was! Appropriately enough the organizers had brought together an impressive number of the pioneers in microscopy, and Sterling Newberry even prepared a book describing the early days of electron microscopy and a video in which said pioneers reminisce about how things were.

How are things now? Very exciting indeed! Many new microscopies have emerged and all fields of microscopy are growing like Topsy. On the way Topsy seems to have been feeding on a very hearty alphabet soup, leaving acronyms as crumbs in its wake. Unfortunately all these little cute short cuts of speech and writing are failly fast tuming (FFT) into big impediments to communication. No one really had problems with EM, SEM and STEM. They sounded like magic incantations. One could clear one's throat by speaking of HREM, or HRSEM, but what to do with HRLVSEM? Everyone got used to Normarski=DIC and only a few eyebrows were raised AVEC-Pol. No problem. But consider the situation today. With the greatest of AESe we have been LED to a rather SED state of affairs. By now I find it hard to RHEED a paper without a glossary (if you need one too, see below). I guess the IMFPs are to blame. The other day I came across TED and SAM and thought it odd that coauthors of the paper would be referred to by first name. It turned out to mean Transmission Electron Diffraction and Scanning Auger Microscopy. I was LED to ponder if these approaches would be better than EFTEM? or ESEM? Actually I like EELS best, especially the way they prepare them in Kyoto. SAM and TED also seem to use an STC where I use a specimen transfer chamber. As we say in French "Quel CHI CHIs"!

Before the main meeting in downtown Boston Linn Hobbs of MIT, with the help of Polaroid, had organized a meeting on "Future Directions in Microscopy \& Imaging" in Southboro, which tums out to be off most MAPS (Microtubule Associated Proteins), but that is another story. I was totally spLEED and in fact somewhat SICMed by the FEGs, TOFSIMS and UVPEMs and had to take to CBED. On reflection (REM) it SIMS that acronyms saved time and, when combined with very long sessions and draconian session leaders, allowed us to survey almost all of microscopy. The problem with the acronyms is that they only allow those people already familiar with a particular field to get much from what is being SED. I tried to relax by taking a cooled CCD but still ended up with NSOMnia and a PMT like state tying to FEG it all out, including PTM. Who knows where it will all LEED?

Many of the acronyms are PIXE-ish, and I am sure put a gleam (not a GLEMA) in the perpetrators eye, a bit like the messages on automobile vanity plates. They also help to sort who is with it and who is not. Many acronyms are useful as they save space, and therefore trees. But abbreviations should not be used at the expense of clarity. They are too often used when not needed. A recent paper I saw introduced the concept of QF-DERS (Quick Freeze, Deep Etch, Rotary Shadow) never to use it again, choosing wisely to spell out quick freeze, deep etch, rotary shadowing, when called for in the body of the paper. A paper is a way to try to communicate with others, not to hide what one has done. If that takes a few more pieces of wood, that might be better than the forest needed to print an encyclopedia to explain the space saving tongue and mind twisting acronyms.

Enough of this. I think I'Il go home now and take a PIMS with a twist of orange PEELS, and let OSA come after Topsy for endangering our collective mental health. May the Force be with you, the AFM of course, oOPC! I meant to say SFM, natch!

\section{- GLOSSARY -}

Many of the acronyms below are found in "Microscopy, The Key Research Tool", ISSN-0146-6119 Published by EMSA, 1992.

AES: Auger Electron Spectroscopy.

AFM: Atomic Force Microscopy

AVEC-POL: Allen Video Enhanced Contrast Polarization microscopy CBED: I saw that somewhere but can't remember what it means; I favor Confocal Ballistic Electron Dispersion, but it is more likely a prosaic "Convergent Beam Electron Diffraction".

CCD: Charge Coupled Device.

$\mathrm{CHI}$ : Concentration Histogram Image.

CHls: Concentration Concentration Histograms.

DIC: Differential Interference Contrast.

EELS: Electron Energy Loss Spectroscopy.

EFTEM: Energy Filtered TEM.

ESEM: Environmental Scanning Electron Microscope.

FFT: Fast Fourier Transform.

GLEMA: Great Lakes EM Association.

IMFP: Inelastic Mean Free Path.

LEED: Low Energy Electron Diffraction.

NSOM: Near-field Scanning Optical Microscope.

MIT: Massachusetts Institute of Technology.

OPC: Oxygen Plasma Clelaning.

OSA: Optical Specimen Annealing.

PEELS: Parallel Electron Energy Loss Spectroscopy.

PIMS: Precision lon Mill System.

PIXE: Proton Induced X-Ray Emission.

PMT: Photo Multiplier Tube.

PTM: Photon Tunnelling Microscope.

RHEED: Reflection High Energy Electron Diffraction.

SED: Secondary Electron Detector.

SFM: Scanning Force Microscopy.

SICM: Scanning lon Conductance Microscopy.

SIMS: Scanning lon Mass Spectroscopy

TOFSIMS: Time of flight SIMS.

UVPEMS: Ultraviolet Photo Electron Microscope.

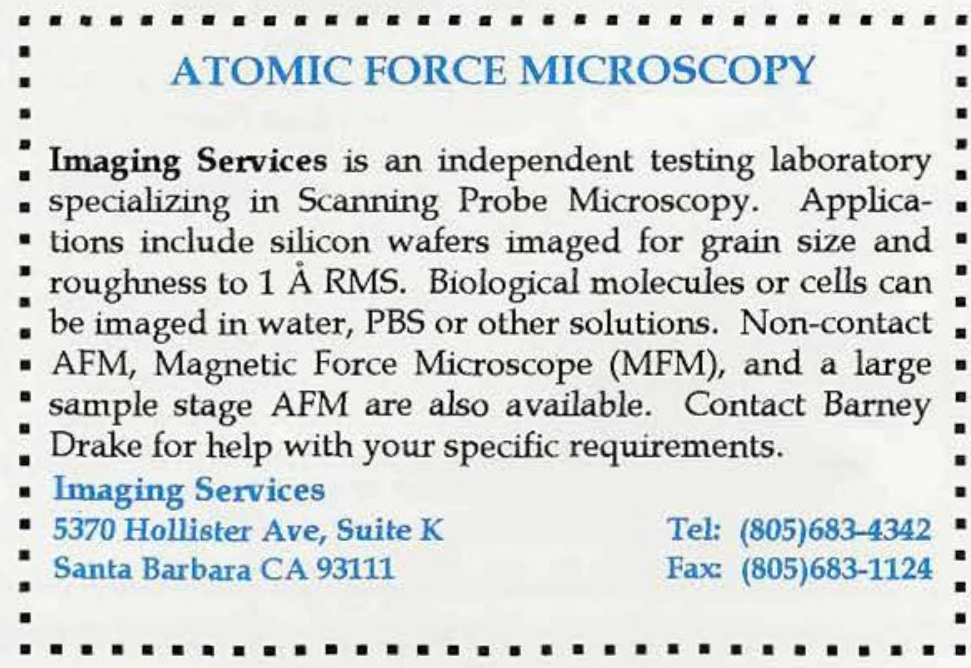

